\title{
A Road Towards the Photonic Hardware Implementation of Artificial Cognitive Circuits

\author{
Eugenio Fazio*
}

Department of Fundamental and Applied Sciences for Engineering, Sapienza Università di Roma via A. Scarpa 16, 00161 Roma Italy

\section{Article Info}

\section{Article Notes}

Received: July 29, 2018

Accepted: September 24, 2018

\section{${ }^{*}$ Correspondence:}

Dr. Eugenio Fazio, Department of Fundamental and Applied Sciences for Engineering, Sapienza Università di Roma via A. Scarpa 16, 00161 Roma Italy;

Email: eugenio.fazio@uniroma1.it.

(c) 2018 Fazio $E$. This article is distributed under the terms of the Creative Commons Attribution 4.0 International License.

\section{Keywords}

Cognitive optics

Reinforcement learning

Smart circuits

Nature mimics

Neural hardware

Photonics

Nonlinear optics

Soliton waves

Solitonic circuits

Stigmergy

Stigmergic circuits

\section{ABSTRACT}

Many technologies we use are inspired by nature. This happens in different domains, ranging from mechanics to optics to computer sciences. Nature has incredible potentialities that man still does not know or that he striving to learn through experience. These potentialities concern the ability to solve complex problems through approaches of various types of distributed intelligence. In fact, there are forms of intelligence in nature that differ from that of man, but are nevertheless exceedingly efficient. Man has often used as a model those forms of distributed intelligence that allow colonies of animals to develop places of housing or collective behaviors of extreme complexity. Recently, M. Alonzo et alii (Sci.Rep. 8, 5716 (2018)) published a hardware implementation to solve complex routing problems in modern information networks by exploiting the immense possibilities offered by light. This article presents an addressable photonic circuit based on the decision-making processes of ant colonies looking for food. When ants search for food, they modify their surroundings by leaving traces of pheromone, which may be reinforced and function as a type of path marker for when food has been found. This process is based on stigmergy, or the modification of the environment to implement distributed decisionmaking processes. The photonic hardware implementation that this work proposes is a photonic $\mathrm{X}$-junction that simulates this stigmergic procedure. The experimental implementation is based on the use of non-linear substrates, i.e. materials that can be modified by light, simulating the modification induced by the ants on the surrounding environment when they leave the pheromone traces. Here, two laser beams generate two crossing channels in which the index of refraction is increased with respect to the whole substrate. These channels act as integrated waveguides (almost self-written optical fibers) within which optical information can be propagated (as happens for the ants that follow traces of pheromone already "written"). The proposed device is a $\mathrm{X}$-junction with two crossing waveguides, whose refractive index contrast is defined by the intensities of the writing light beams. The higher the writing intensity, the greater the induced index variation, as if it were an increasingly intense pheromone trace. The information will follow the most contrasted harm of the junction, which is driven and eventually switched by the writing light intensity. Any optical information that will be sent to the device will follow the most intense trace, i.e. the most contrasted waveguide. The paper demonstrates a device that can be wholly operated using the light and that can be the basis of complex hardware configurations that might reproduce the stigmergic distributed intelligence. This is a highly significant innovation in the field of electronic and photonic technologies, within which artificial cognition and decision processes are implemented into a hardware circuit and not in a software code.

\section{Introduction}

Men created a wonderful technological world, full of smart solutions and possibilities. However, as our own knowledge and technological innovations develop, we are ever-discovering that 
nature itself possesses and uses very sophisticated technologies beyond our current scientific comprehension. In all fields, nature is in itself hyper-perfected: nothing is left to chance, everything is adapted and optimized to perform its function at its best. Many of the new technologies invented by man are inspired by nature, observing and reproducing it in function and structure. A typical example is the "Velcro", the tear-off tissue that can easily be hooked and unhooked, which is inspired by the way burdocks (alpine plants) attach themselves to the animal's skin or fur in order to spread their seeds (the Velcro was invented by the Swiss engineer George de Maestral in 1941 following his observations on these mountain flowers). Men have learned to increase the ability of adhesion between surfaces through micro- and nanostructuration, by copying the fingerprints of the hands or the fine hairs that the geckos ${ }^{1}$ have on the fingertips of the feet, exploiting Van der Walls electric forces and managing to overcome gravity. The wonders of nature's ingenuity are not limited to mechanics ${ }^{2}$. Indeed, nature perpetuates itself in various capacities and with methods and biological designs that we would never have imagined existed until our recent technological developments have allowed us to do so. For example, it was extraordinary to discover that nature uses light to communicate and transfer information, just as man did first with Morse language and, later on, with fiber optic communications. In the universe, light has three specific functionalities: it transports energy, it transports information, and it signals the passage of time. All this would seem normal: and yet the functions of light extend beyond this. In fact, light is used at the cellular or embryonic level to carry information, energy and perpetuate synchronization. Seeds of different plants (for example lentils or beans ...) emit light due to biochemical reactions during both dormancy and germination ${ }^{3}$. This level of emitted light used at the cellular and embryonic levels is so slight that we cannot observe its presence with the naked eye, but it is nevertheless present. Initially it was thought that this biochemical luminescence was absolutely random, uncorrelated with any vital process. Recent study ${ }^{4}$, however, has found that the seed coat is a true sensorial station of the external environmental conditions, which emits in the form of light (electromagnetic signaling) an entire series of important information for its germination. This luminous information is gathered directly from the seed's coat, and also acts as an internal reflector. In fact, the shape of the seeds are not random; they are perfect luminous collectors that maintain light emission and redirect this light on exactly the embryo plumula ${ }^{5}$ (figure 1 ). It is a masterly system, an optimized, functionalized biomorphism, able to make the most of its structural potentialities with the aim of creating a new plant.

The systems and techniques that nature possesses and exploits to solve problems and optimize processes

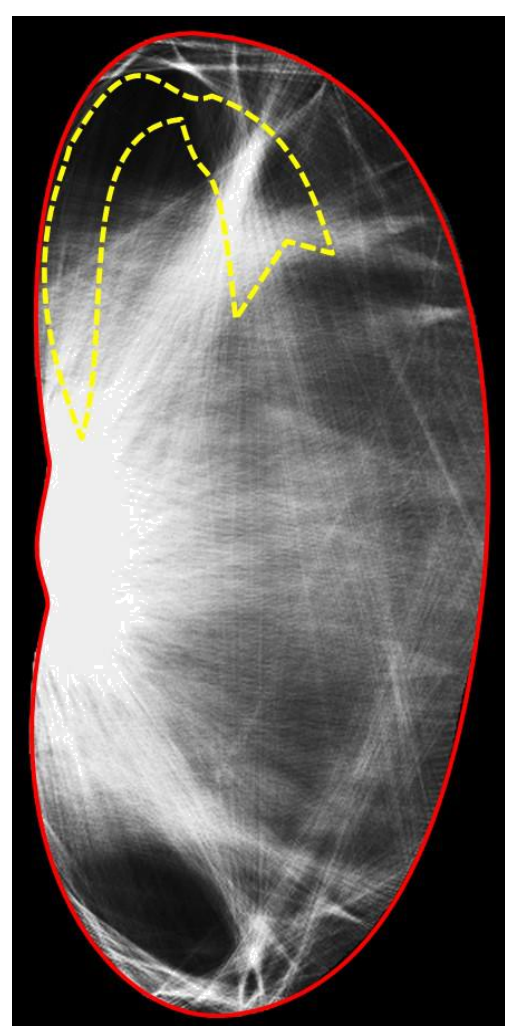

Figure 1: Ray tracing of light inside a bean-shaped reflecting cavity.

also operate at higher levels in that they also concern the cognitive capacity of nature. I do not speak of the intelligence of individual animals or single plants ${ }^{6}$, but of complex systems such as colonies of insects, flocks of birds, or herds of animals. There is a form of distributed intelligence that can cope with complex problems that our own mathematics cannot solve in a simple way. Modern computer-network software often uses algorithms inspired by nature. For example, I refer to all those protocols for the optimization of multi-variable problems known as genetic algorithms, which exploit the rules of genetics to solve mathematical problems with many independent variables, or neural networks, mathematical systems that base the calculation on a "learning" database that the system has previously prepared ${ }^{7,8}$.

Many topological problems, such as routing in a computer network, are often analyzed using protocols inspired by how animal colonies solve practical problems, such as the search for food by ants ${ }^{9,10}$. It is precisely this problem, also called the travelling salesman problem ${ }^{11}$, that has been widely studied in the past as a possible solution to problems of information routing in complex computer networks. It is a typical problem that would require a smart signal processing, which is called "reinforcement learning".

This expression is commonly used in computer science to describe those algorithms "of machine learning inspired by behaviorist psychology, concerned with how software 
agents ought to take actions in an environment so as to maximize some notion of cumulative reward ${ }^{12}$."

Reinforcement learning regards neural networks or artificial intelligence protocols that self-set by reinforcing a specific information identified by a feedback in the system in order to solve complex problems. This procedure is indeed inspired by nature, adopting its stigmergy in order to transfer information in decentralized systems, thus realizing distributed cognitive processes through many small, simple elaborations. The term stigmergy describes precisely that form of communication based on the modification of the surrounding environment. Unfortunately, stigmergic software protocols need solution times that increase exponentially with the size of the problem; after many years of research, no improved algorithm has been found to solve these problems within a polynomial time using a deterministic Turing machine. For this reason, hardware approaches have been proposed in the past ${ }^{13-18}$; among all, optical solutions to supercomputing seem to win for versatility ${ }^{19}$ in terms of increased fanin and fan-out, energy-consumption, and recursive preprocessing. However, the proposed optical solutions ${ }^{20-26}$ neither reduce the complexity of the problem nor offer technologically efficient procedures without exponentially increasing the demand of physical resources ${ }^{27,28}$.

Very recently an alternative approach was proposed to realize a photonic hardware able to perfectly simulate the stigmergic processes adopted by ants searching for food (the salesman problem solver). This alternative approach was published in the paper M. Alonzo, D. Moscatelli, L. Bastiani, A. Belardini, C. Soci and E. Fazio titled All-Optical
Reinforcement Learning In Solitonic X-Junctions by $\mathrm{M}$. Alonzo, D. Moscatelli, L. Bastiani, A. Belardini, C. Soci and E. Fazio ${ }^{29}$.

\section{Methodology}

Let's see how it works. When ants go looking for food, each ant follows a random path, highlighted by the emission of a pheromone trace (figure 2). If the food is found, the right path is reinforced with pheromone on the way back to the anthill. Each other ant crossing such strengthened trace recognizes this path as the one to the food and starts following it, reinforcing it as well. After some time, all the other pheromone traces vanish, leaving active just the boosted ones. It is possible to reproduce the ant's decisional procedure in a photonic system by adopting an optical substrate that can be modified by the light propagating inside. This can be obtained using specific nonlinear media through host materials, such as the electro-optic photorefractive ones, which are the ideal stigmergic environment for actively addressable photonics.

The processing capacity of the ant's colony is based on two simple decision-making processes: the first concerns the followed trajectory, which can be reinforced or annihilated according to a precise external feedback (which, for the ants, is represented by the quantity of deposited pheromone); the second concerns the chosen direction in a node point, which is is at the intersection of two trajectories (the ants will channel into the trajectory with a more intense pheromone trace).

Both processes imply nonlinear responses to the feedback, which can be implemented using nonlinear

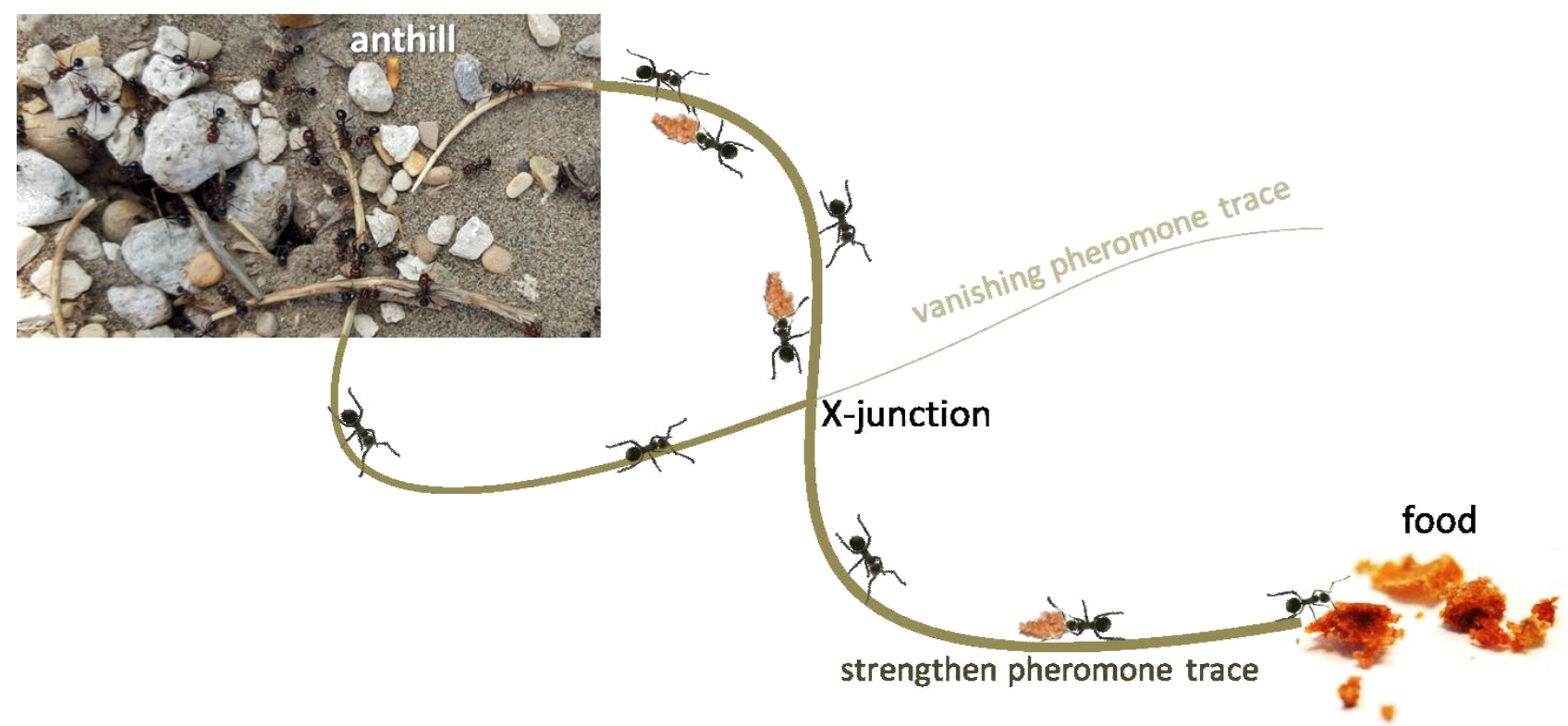

Figure 2: Basic scheme of the search of food by the ants. The system is based on the two fundamental decision-making principles of following a trace of pheromore and of changing track when a more marked one is met 
optical materials whose refractive index can be modulated by optical stimuli. In the work by Alonzo et alii the pheromone trajectories are represented by paths of modified refractive index of the host material. When a laser beam is injected inside a photorefractive medium, the light absorption generates free or trapped charges according to both the structure of the host material and the photon energy. Trapped charges can locally modulate the refractive index via the electro-optic effect, acting as writing tools for channels where the refractive index is increased compared to the surrounding environment Such modifications behave as induced waveguides, i.e. regions that confine optical information which can travel inside them without being dispersed (as signals in optical fibers). The refractive contrast between the induced channel and the surrounding medium depends on the intensity of the writing beam. Consequently, it behaves like the pheromone quantity in the ant's path: it can be strengthen of weakened with the writing light intensity.....until the complete deletion. The second decision-making process is represented by a nonlinear modulation of the crossing point between these paths. The strengthening of one path in a X-crossing point would correspond to making it a preferential trajectory, where the light will be conveyed more easily. It behaves as a channel of water whose banks have been made deeper and therefore more capacious: when two channels meet, more water will flow into the deepest channel rather than inside the shallowest one. Such addressable behavior has been induced into a nonlinear optical X-junction. The junction has been realized by injecting two absorbed beams that cross one each other in the middle of the host photorefractive medium. Each beam modulated the refractive index of the host medium according to its own intensity. A third (unable to modify the host medium) is injected inside one channel and consequently reaches the $\mathrm{X}$ junction. It represents the information that propagates inside the photonic structure. If the writing beams have the same intensities, the junction is perfectly symmetrical, meaning that $50 \%$ of the information beam emerges from one channel and $50 \%$ from the other one (figure 3 ). When the writing light is unbalanced or a writing feedback is injected from the output, the X-junction switches to an asymmetric behavior, for which $80 \%$ of the information beam is now conveyed inside the strengthened channel and the remaining $20 \%$ remains in the weaker one.

\section{Summary}

Such configuration shows a significant advantage with respect to the most traditional optical circuits where all the light trajectories were previously artificially realized and fixed. In the case of this study, the entire structures are able to be both induced and erased by light. It is a fully addressable configuration. Thus, this new approach opens important perspectives for realizing circuits able

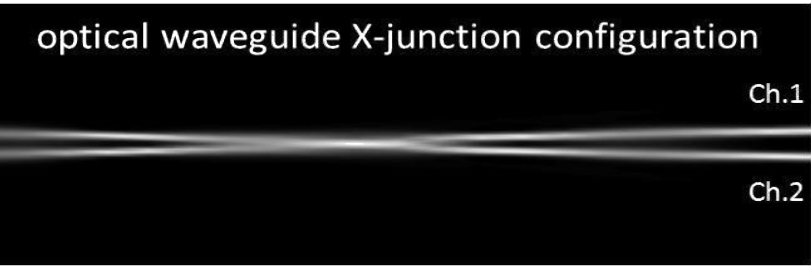

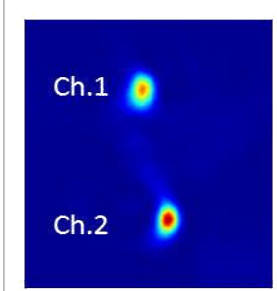

waveguides output profile

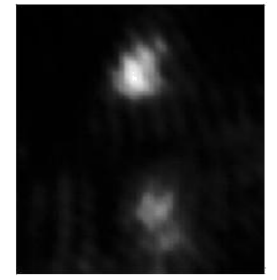

signals output without feedback

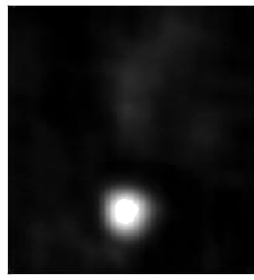

signals output with feedback
Figure 3: Numerical simulation (top) and experimental results (below) of a stigmergic photonic $x$-junction.

to mimic intelligent circuits with geometries similar to neural systems. It would be possible to realize hardware neural circuits using light to transmit information, energy, and a temporal trigger. The adoption of light for signal processing and cognitive systems is an ambitious goal that has long been sought after ${ }^{30-32}$, but it is only in recent study that important advances have been made toward its real, practical implementation. Will the optical computer be realized, or will it remain a dream of science fiction movies? In light of this question, many may recall the Hal 9000 computer inside the Discovery spaceship featured in Stanley Kubrick's iconic film 2001: A Space Odyssey. In perhaps the most pivotal and visionary scene in the film, astronaut Bowman floats into the interior compartment of memory banks in the over-intelligent, over-autotomized Hal in order to exterminate the system, the computer all the while trying to dissuade him. In the red-lit chamber, Bowman dislodges a series of light-filled memory banks of transparent and luminous crystals. With the findings of this study and the potential for further research and discoveries it presents in mind, do we conceive of this scene as only the brilliant vision of a science fiction screenwriter or the premonition of a near future of practical technology?

\section{Acknowledgement}

The author acknowledges S. Bevacqua Collins for a critical reading of the manuscript.

\section{References}

1. Autumn K, Liang YA, Hsieh ST, et al. Full, Adhesive force of a single gecko foot-hair. Nature. 2000; 405: 681-685. DOI: 10.1038/35015073

2. Fratzl P. Biomimetic materials research: what can we really learn from nature's structural materials. Journal of Royal Society Interface. 2007; 4: 637-642. DOI: $10.1098 /$ rsif.2007.0218

3. For a complete review see the book Recent Advances in Biophoton 
Research and its Application, ed. By FA Popp, KH Li, Q Gu, World Scientific, Singapore. 1992. DOI: 10.1142/1559

4. Footitt S, Palleschi S, Fazio E, et al. Ultraweak Photon Emission from the Seed Coat in Response to Temperature and Humidity - A Potential Mechanism for Environmental Signal Transduction in the Soil Seed Bank. Photochemistry and Photobiology. 2016; 92: 678-687. DOI: 10.1111/php.12616

5. Fazio E, Gualandi G, Palleschi S, et al. Optically functionalized biomorphism of bean seeds. Journal of Luminescence. 2017; 182C: 189-195. DOI: 10.1016/j.jlumin.2016.10.034

6. Stefano Mancuso, Alessandra Viola. Brilliant Green: The Surprising History and Science of Plant Intelligence. Island Press. 2015. DOI: $10.1086 / 685354$

7. Zamanisabzi H, King JP, Dilekli N, et al. Developing an ANN Based Streamflow Forecast Model Utilizing Data-Mining Techniques to Improve Reservoir Streamflow Prediction Accuracy: A Case Study. Civil Engineering Journal. 2018; 4: 1135-1156. DOI: 10.28991/cej0309163

8. Saljoughi AS, Mehrvarz M, Mirvaziri H. Attacks and Intrusion Detection in Cloud Computing Using Neural Networks and Particle Swarm Optimization Algorithms. Emerging Science Journal. 2017; 1: 179-191. DOI: $10.28991 / \mathrm{ijse}-01120$

9. Goss S, Aron, JL Deneubourg JM . Pasteels, Self-organized shortcuts in the Argentine ant. Naturwissenschaften. 1989; 76: 579-581. DOI: $10.1007 / \mathrm{BF} 00462870$

10. Deneubourg JL, Aron S. Goss JM. Pasteels, The self-organizing exploratory pattern of the Argentine ant. J Insect Behav. 1990; 3: 159. DOI: $10.1007 / \mathrm{BF} 01417909$

11. Bentley JL. Fast algorithms for geometric traveling salesman problems. ORSA J Comput. 1992; 4: 387-411. DOI: 10.1287/ijoc.4.4.387

12. "Reinforcement Learning", at https://en.wikipedia.org/wiki/ Reinforcement_learning, published February 28, 2018.

13. Aaronson S. NP-complete problems and physical reality. ACM SIGACT News. 2005; 36: 30-52. DOI: 10.1145/1052796.1052804

14. Kieu T. Quantum algorithm for Hilbert's tenth problem. Int J Theor Phys. 2003; 42: 1461-1478. DOI: 10.1023/A:1025780028846

15. O’Brien JL. Optical quantum computing. Science. 2007; 318: 15671570. DOI: $10.1126 /$ science. 1142892

16. Adleman LM. Molecular computation of solutions to combinatorial problems. Science. 1994; 266: 1021-1024. DOI: 10.1126/ science.7973651
17. Ezziane Z. DNA computing: applications and challenges. Nanotech. 2006; 17: R27. DOI: 10.1088/0957-4484/17/2/R01

18. Liu Q, Wang L, Frutos AG, et al. DNA computing on surfaces. Nature. 2000; 403: 175-179. DOI: 10.1038/35003155

19. Caulfield HJ, Dolev S. Why future supercomputing requires optics. Nat Photon. 2010; 4: 261-263. DOI: 10.1038/nphoton.2010.94

20. Oltean M. Solving the Hamiltonian path problem with a light-based computer. Nat Comput. 2008; 7: 57-70. DOI: 10.1007/s11047-0079042-z

21. Shaked NT, Messika S, Dolev S, et al. Optical solution for bounded NP-complete problems. Appl Opt. 2007; 46: 711-724. DOI: 10.1364/ A0.46.000711

22. Woods D, Naughton TJ. Optical computing: photonic neural networks. Nat Phys. 2012; 8: 257-259. DOI: 10.1038/nphys2283

23. Haist T, Osten W. An optical solution for the traveling salesman problem. Opt Express. 2007; 15: 10473-10482. DOI: 10.1364/ OE.15.010473

24. Sartakhti JS, Jalili S, Rudi AG. A new light-based solution to the Hamiltonian path problem. Future Gen Comput Syst. 2013; 29: 520 527. DOI: $10.1016 /$ j.future.2012.07.008

25. Dolev S, Fitoussi H. Masking traveling beams: optical solutions for NPcomplete problems, trading space for time. Theor Comput Sci. 2010; 411: 837-853. DOI: 10.1016/j.tcs.2009.06.030

26. Goliaei S, Jalili S, Salimi J. Light-based solution for the dominating set problem. Appl Opt. 2012; 51: 6979-6983. DOI: 10.1364/ A0.51.006979

27. Wu K, Garcia de Abajo J, Soci C, et al. An optical fiber network oracle for NP-complete problems. Light Sci \& Appl. 2014; 3: e147. DOI: 10.1038/lsa.2014.28

28. Hu W, Wu K, Shum PP, et al. All-optical implementation of the ant colony optimization algorithm. Sci Rep. 2016; 6: 26283. DOI: 10.1038/ srep26283

29. Alonzo M, Moscatelli D, Bastiani L, et al. All-Optical Reinforcement Learning In Solitonic X-Junctions. Sci Rep. 2018; 8: 5716. DOI: 10.1038/s41598-018-24084-w

30. Jahns J. Concepts of optical digital computing-A survey. Optik. 1980; 57: 429-449.

31. Sawchuk AA, Strand TC. Digital optical computing. Proc IEEE. 1984; 72: 758 - 779. DOI: 10.1109/PROC.1984.12937

32. Woods D, Naughton TJ. Optical computing: Photonic neural networks. Nature Physics. 2012; 8: 257-259. DOI: 10.1038/nphys2283 